\title{
Computerized Tomography-Guided Core-Needle Biopsy of Orbital Space-Occupying Lesions: A Case Series
}

\author{
Bashar M. Bata ${ }^{a}$ Andrew Martin ${ }^{b}$ Daniel Connolly ${ }^{b}$ Hardeep Singh Mudhar ${ }^{c}$ \\ Naomi Hersey ${ }^{b}$ Sachin M. Salvi ${ }^{a}$ \\ a Department of Ophthalmology, Royal Hallamshire Hospital, Sheffield, UK; ${ }^{b}$ Department of Radiology, Royal \\ Hallamshire Hospital, Sheffield, UK; ' National Specialist Ophthalmic Pathology Service (NSOPS) Department of \\ Histopathology, Royal Hallamshire Hospital, Sheffield, UK
}

\section{Keywords}

Orbital biopsy $\cdot$ CT guidance $\cdot$ Orbital tumours

\begin{abstract}
Purpose: To describe our experience in performing biopsy of post-septal orbital masses with core needle under computerized tomography guidance (CT-CNB). Methods: The medical records of all patients who underwent this procedure were reviewed. The procedure was performed under local anesthesia on a day case basis under a peribulbar block. A planning non-contrast computerized tomography (CT) scan of the orbits was performed to localise the mass. A 6-cm 18-G Temno Evolution ${ }^{\circledR}$ semi-automated biopsy needle was inserted through the skin into the orbit. Prior to further advancement of the needle, a low-dose CT limited to the previously determined plane was performed to confirm its position. The needle was then advanced, and the cutting needle was deployed to obtain the biopsy. Results: Five patients who underwent CT-CNB were identified. The CNB was successful in 4 patients and revealed a metastatic prostate adenocarcinoma, diffuse large B-cell lymphoma, a metastatic neuroendocrine tumour, and orbital inflammatory disease. The biopsy failed in the fifth patient when the needle failed
\end{abstract}

to penetrate the tumour despite good localisation on CT. He was eventually diagnosed with fibrous meningioma of the greater wing of sphenoid on open biopsy. None of the patients had any complications other than peri-ocular bruising which was present in all of them. Conclusion: CT-CNB of mass lesions located in the lateral aspect of the orbit can be an alternative to open biopsy in selected cases. It avoids major surgery and allows the use of radiotherapy, if required, without any delay.

(c) 2020 S. Karger AG, Basel

\section{Introduction}

Space-occupying lesions in the orbit can be of varied aetiologies. Many present with similar features, making them difficult to distinguish clinically and radiologically. Hence, a biopsy is often required to make an accurate diagnosis and then deliver correct therapy. The biopsy is typically obtained surgically via an orbitotomy, which often is a major surgical procedure that requires general anaesthesia. An orbitotomy may not be feasible in elderly, severely ill patients, and is associated with high morbidity. Moreover, in patients in whom radiotherapy is re-

$\begin{aligned} & \text { karger@karger.com } \\ & \text { www.karger.com/oop }\end{aligned}$
Karger $\%$

Bashar M. Bata

Department of Ophthalmology, Royal Hallamshire Hospital Glossop Rd

Sheffield, S10 2JF (UK)

drbata@doctors.net.uk 

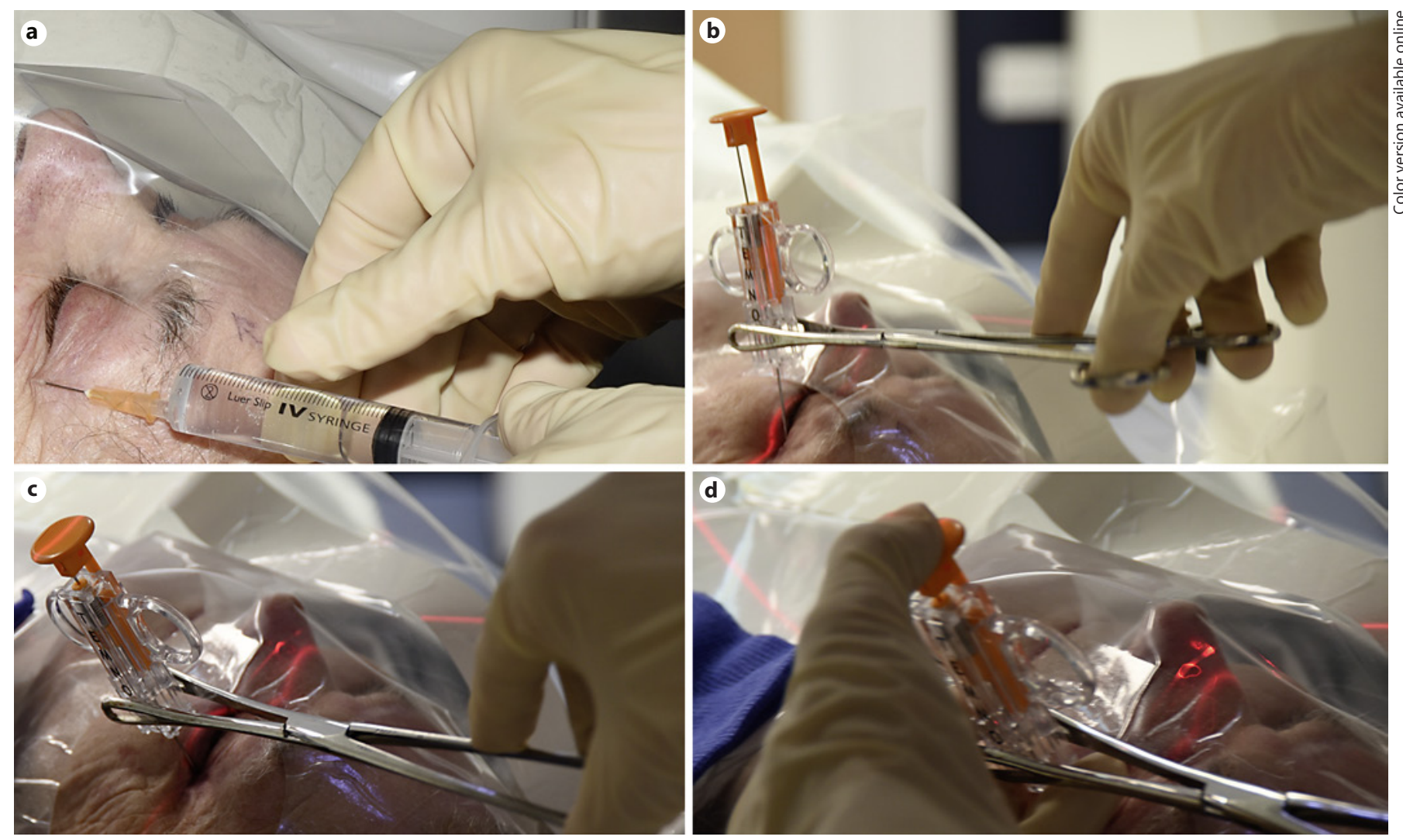

Fig. 1. The steps of CT-CNB. a Local anesthesia. b CT scan being performed while the needle is stabilised using Rampley sponge forceps. c The needle trajectory is adjusted. $\mathbf{d}$ Deployment of the cutting needle.

quired, it causes a delay of at least 6 weeks to allow for healing of the surgical site.

Fine-needle aspiration (FNA) with and without image guidance has been used to sample orbital lesions as a less invasive alternative to conventional surgery [1-6]. However, in a study by Krohel et al. [7], FNA of space-occupying lesions of the orbit was inaccurate or insufficient to make a diagnosis in $>50 \%$ of patients. This inaccuracy was attributed to the fact that FNA alters the tissue architecture and is only suitable for a cytological analysis.

Core-needle biopsy $(\mathrm{CNB})$ is another minimally invasive alternative to open biopsy that is widely used to sample tumours throughout the body. CNB usually has a greater diagnostic accuracy and provides more tissue than FNA, allowing for detailed histopathological assessment, immunophenotyping, and electron microscopy [8-10]. CNB of orbital lesions has previously been performed blindly or under ultrasound guidance, both of which are more suitable for palpable lesions that lie anteriorly in the orbit [11-13]. For deeper retrobulbar lesions, however, it is technically more difficult and there is a greater risk of injury to the globe, optic nerve, extraocular muscles, and vascular structures. Performing CNB under CT guidance can potentially lower the risk of complications and allow more accurate sampling of orbital pathology. There are only 2 reports on small-scale studies of CT being used to acquire $\mathrm{CNB}$ of space-occupying lesions of the orbit $[14,15]$. The purpose of this study was to describe our experience in performing CT-guided CNB (CT-CNB) of orbital lesions.

\section{Methods}

We reviewed the medical records of all patients who had undergone CT-CNB of orbital space-occupying lesions at a tertiary referral centre in Sheffield, UK. Information collected included: age, gender, previous medical history, presenting symptoms, clinical signs, radiographic features, indication for biopsy, and histopathological diagnosis. 

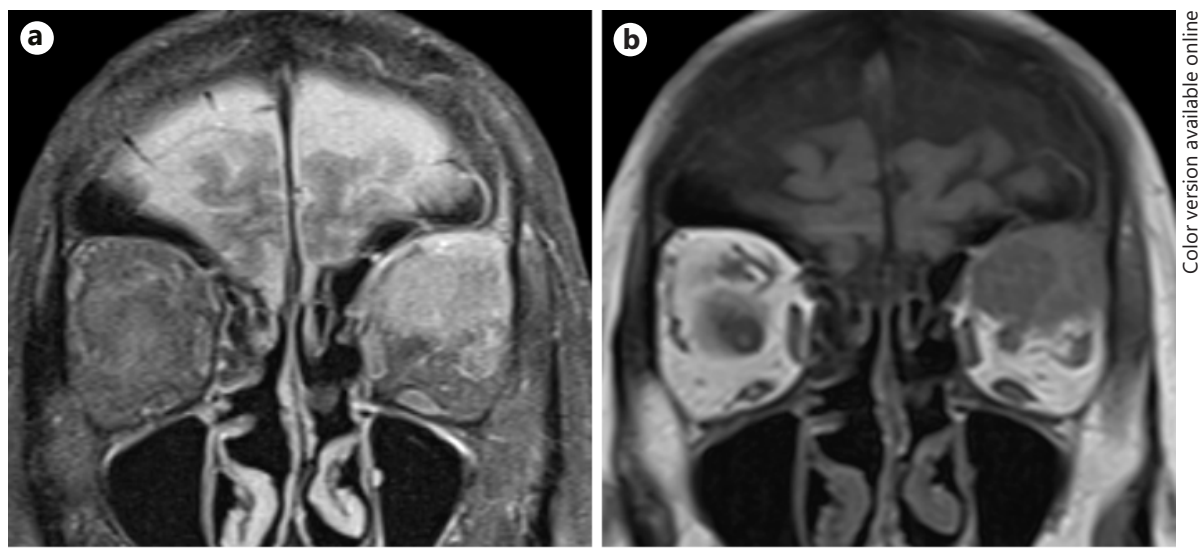

Fig. 2. Case 1. a, b Selected images of the orbits. a Fat-saturated coronal T2. b Fatsaturated coronal T1 demonstrating a hyperintense-to-muscle (T2) and isointenseto-muscle (T1) mass lesion within the superior and lateral aspect of the left orbit with associated proptosis of the globe. c, d Selected axial images of a non-contrast $\mathrm{CT}$, demonstrating the level at which we targeted the retro-orbital mass (c) and the biopsy needle in place before deployment of the cutting needle (d). e Staining shows atypical lymphoid cells. H\&E. f Immunohistochemistry shows brown positive staining for CD20 indicating a B-cell lymphoma. The morphology and further immunohistochemical panels (not shown) indicated the diagnosis of diffuse large B-cell lymphoma (DLBCL).
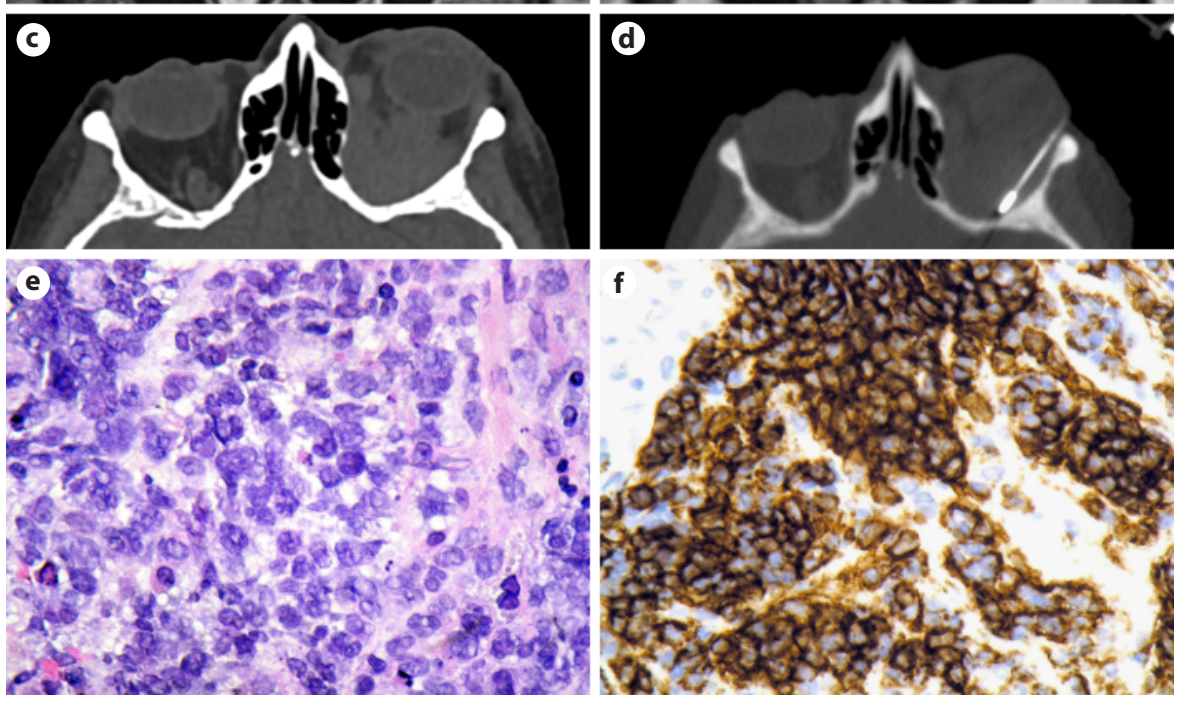

\section{Technique}

The procedure was performed under local anaesthesia on a day case basis. A peribulbar block (a combination of $5 \mathrm{~mL}$ of $2 \%$ Lidocaine, 1:200,000 adrenaline, $5 \mathrm{~mL}$ of $0.5 \%$ bupivacaine, and 1,500 IU hyaluronidase) was administered in the anaesthetic room in the CT scan suite by the ophthalmology team approximately 15 min prior to the procedure. Further top-up local anaesthetic mixture was administered as necessary until total akinesia was achieved.

The patient was then transferred to the CT scanner (Cannon Aquilion Prime) and positioned supine and head-first on the CT table. An initial non-contrast CT was performed through the orbits to allow localisation of the mass. The images were reviewed, and the optimal axial CT slice providing the safest access into the mass was selected. A reference light was used to illuminate the plane of the selected slice, and an entry point at the inner table of the lateral orbital wall was chosen to gain access to the lateral orbit.

One non-vascular interventional radiologist (Consultant A) performing the procedure, was positioned behind the gantry of the $\mathrm{CT}$ scanner, and the other (Consultant B) viewed the imaging on the monitor next to the CT gantry and gave verbal instructions regarding needle position. This setting was necessary as one radiologist could not perform the biopsy and view the screen simultaneously due to positioning of screen in relation to procedure site. The treating orbital surgeon was also present throughout the pro- cedure to perform a canthotomy and cantholysis in the event of retrobulbar haemorrhage.

The involved eye was prepped with $10 \%$ aqueous Betadine and draped to create a sterile field. Local anaesthetic ( $1 \mathrm{~mL}$ of $2 \%$ Lidocaine with adrenaline) was injected subcutaneously at the lateral canthus (Fig. 1a) and a small skin incision was made at the selected point of entry. A 6-cm 18-G Temno Evolution ${ }^{\circledR}$ semi-automated biopsy needle, held by a pair of long locking Rampley sponge forceps, was inserted through the skin incision into the orbit (Fig. 1b). The needle was gently advanced manually and directed by hand, keeping it in the same plane as the selected CT slice and so into the mass at the selected safe entry point. The forceps were used to keep the needle in the correct plane, represented by the reference light, whilst further imaging was performed (Fig. 1c). Once inside the orbit and prior to further advancing the needle, a low-dose CT, limited to the previously determined plane, with 1 slice $4 \mathrm{~mm}$ above and 1 slice $4 \mathrm{~mm}$ below, was performed. The position of the needle tip was confirmed to be perpendicular to the chosen plane by confirming that the needle appeared along its length only in the chosen slice and not in the slice above or the slice below; had it been seen in either of these, this would have indicated that the needle was angled cranially or caudally rather than perpendicular. The needle was advanced slowly into the orbit with the limited CT scan repeated every few millimetres or when need- 
Fig. 3. Case 2. a, b Selected images of the orbits. a Coronal T2 fat-saturated postgadolinium. b Axial T1 fat-saturated postcontrast. These demonstrate a right superior and lateral isointense-to-muscle enhancing mass lesion. The mass is apart from the lacrimal gland and extraocular muscles. There is abnormal thickening and signal intensity of the lateral orbit wall. There is evidence of enhancing extradural soft tissue within the right middle cranial fossa (white arrow) and enhancement of the right temporalis muscle (white arrowhead). c, d Selected axial images of a noncontrast CT demonstrating the level at which we targeted the retro-orbital mass (c) and the biopsy needle in place before deployment of the cutting needle (d). This also demonstrates the expanded lateral orbital wall. e Staining shows round tumour cells in a fibrous matrix. Some of the tumour cells are arranged as acini (arranged in circular configurations). H\&E. f Immunohistochemistry shows brown positive staining with prostate specific acid phosphatase, confirming the diagnosis of metastatic prostate adenocarcinoma.
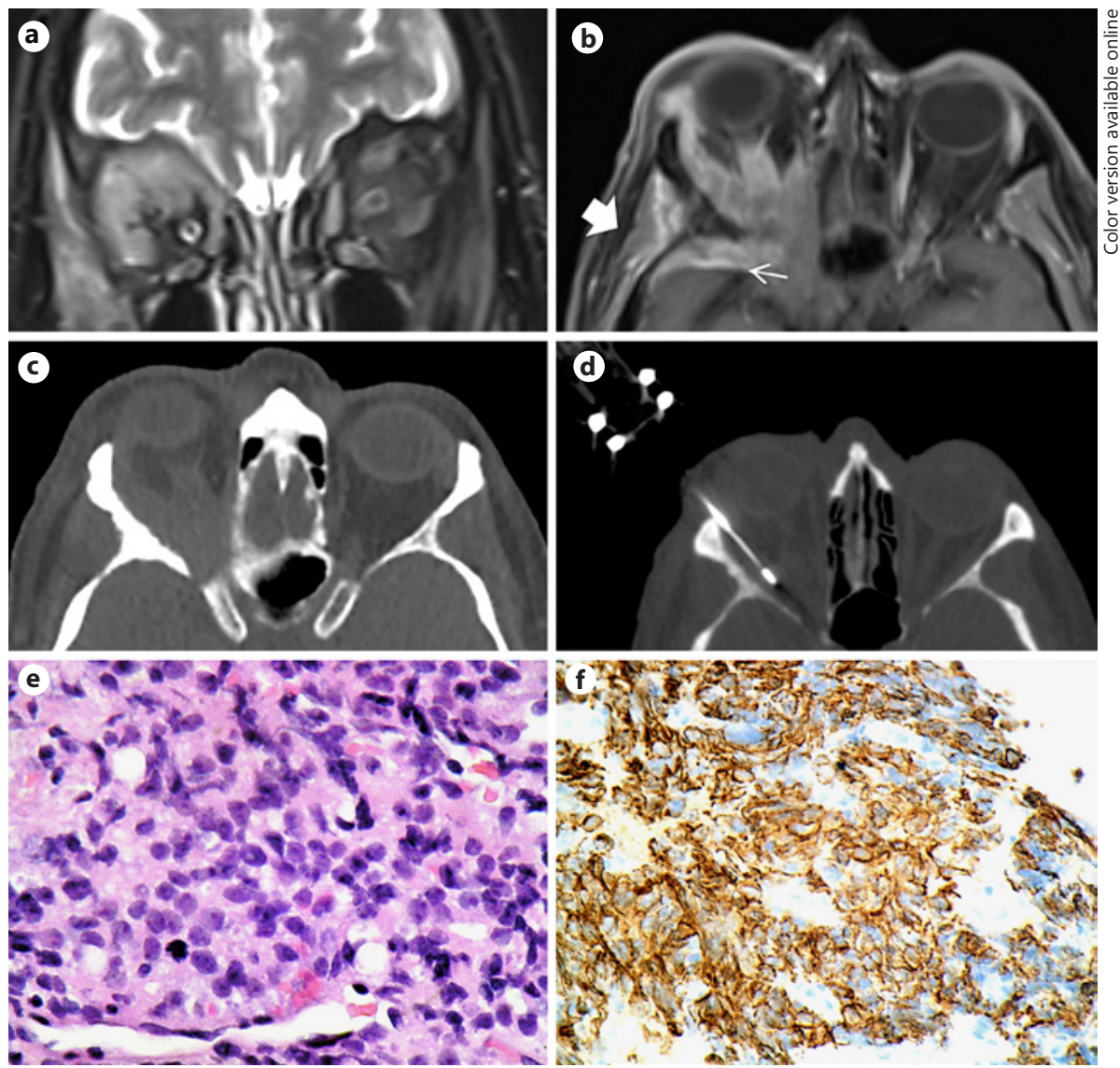

ed. Whilst being advanced, the needle was angled medially or laterally as per the instructions from Consultant B but was kept in the same axial plane throughout the procedure. This continued until the needle tip was at the mass, after which the biopsy stylet was advanced inside the mass. Once in the desired position, the cutting needle was deployed (Fig. 1e). The needle was then removed, and the sample was placed in formalin solution. This process was then repeated, using a fresh needle at each pass, to allow for 2 or 3 samples to be obtained, depending on the ease of access and patient compliance. The specimens were then sent for histopathological analysis.

Chloramphenicol ointment and a pressure dressing were applied to the eye for $2 \mathrm{~h}$ to prevent corneal exposure and retrobulbar haemorrhage. The patient was reviewed $2 \mathrm{~h}$ post-procedure and prior to discharge, to ensure there were no sight-threatening complications. The patient's routine anti-coagulation treatment was commenced the following day as per hospital policy and medical advice.

\section{Results}

Five patients who underwent CNB biopsy under CT guidance over a 12-month period were identified. They all underwent orbital imaging after presenting with features suggestive of orbital inflammatory and/or neoplastic disease or after an episode of loss of consciousness. In all 5 patients, the mass lesion was retrobulbar and occupied the supero-lateral aspect of the orbit. The biopsy needle was introduced through a skin incision made along the lateral orbital rim in all patients. The needle was passed just inside the lateral orbital above or below the lateral rectus muscle.

The CNB was successful in 4 out of 5 patients. Following the biopsy, a diagnosis of metastatic disease was made in 2 patients (prostate adenocarcinoma and poorly differentiated neuro-endocrine tumour) and B-cell lymphoma was diagnosed in 1 patient (Fig. 2-4). In Case 5 (Fig. 5), the biopsy showed a fibroinflammatory process; a diagnosis of inflammatory orbital disease was thus made, and no further biopsies were required. The biopsy in this patient showed skeletal muscle fibres, confirming that the source of the biopsy was the muscle. The patient responded rapidly to systemic steroids, with bilateral improvement of signs and symptoms, and did not show any evidence of recurrence once systemic steroids were stopped. The patient in whom the CNB failed (Fig. 6) eventually underwent a lateral orbitotomy and was diagnosed with 
Fig. 4. Case 3. a Axial non-contrast CT through the orbits, demonstrating a softtissue mass within the greater wing of the left sphenoid bone. The sphenoid wing is expanded with mass effect on the retro-orbital components, displacing the lateral rectus and optic nerve medially. b, c Selected axial MRI images of the orbits: axial T2 (b) and axial T1 post-contrast (c). They demonstrate an isointense-to-muscle mass with homogenous enhancement within the left sphenoid bone, with some adjacent dural enhancement (white arrow). d, e Selected axial images of a non-contrast CT demonstrating advancement of the needle into the mass. f Staining shows a core of blood only, indicating the failure of the biopsy technique to acquire a tissue sample. H\&E.

Fig. 5. Case 5. a, b Selected axial and coronal images from an unenhanced head CT. The scan demonstrates a diffusely abnormal, thickened right lateral rectus muscle with some surrounding inflammatory fatstranding. There is associated thickening of the peri-orbital soft tissues. There is also some minor thickening of the left lateral rectus muscle but not to the same extent. c, d Selected MRI images of the orbits performed 2 days later. c Axial T2 demonstrating thickening of the lateral rectus muscles bilaterally, more pronounced on the right with some associated high T2 signal and an indistinct border to the lateral recti. $\mathbf{d}$ Axial T1 post-gadolinium demonstrating diffuse enhancement of the lateral recti, more pronounced on the right with associated peri-orbital soft tissue enhancement. e Staining shows a fibro-inflammatory process. The fibrous component is eosinophilic (pink) and the inflammatory component is basophilic (blue), punctuating the fibrous tissue. H\&E. f CD68 immunohistochemistry indicates the presence of macrophages.
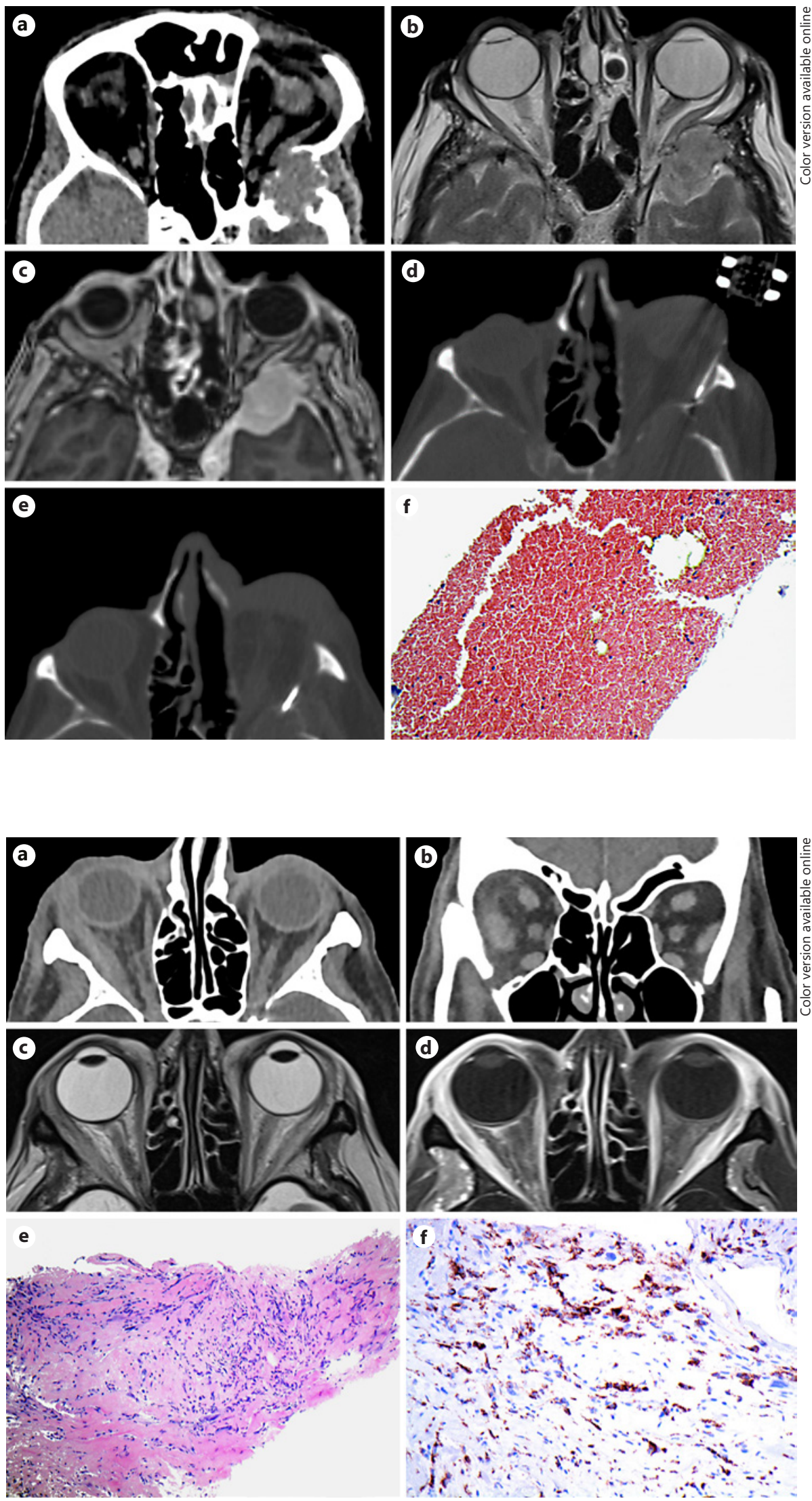

58

Ocul Oncol Pathol 2021;7:54-61

DOI: $10.1159 / 000510867$

Bata/Martin/Connolly/Mudhar/Hersey/ Salvi 
Fig. 6. Case 4. a-c Selected axial images of the orbits: axial T2 (a), coronal T2 (b), and axial T1 (c) post-gadolinium. These demonstrate an isointense-to-muscle homogenously enhancing mass lesion within the left retro-orbital compartment. It is closely associated to the left lateral rectus muscle with no distinct plane seen between the muscle and the mass. d Axial image of a non-contrast CT demonstrating the biopsy needle in place within the mass before deployment of the cutting needle. e Staining shows tumour cells arranged as trabeculae with a suggestion of resetting. The tumour cells are packed close and focally moulding together. H\&E. f Immunohistochemistry shows brown positive staining to synaptophysin, confirming the diagnosis of a metastatic neuroendocrine carcinoma.
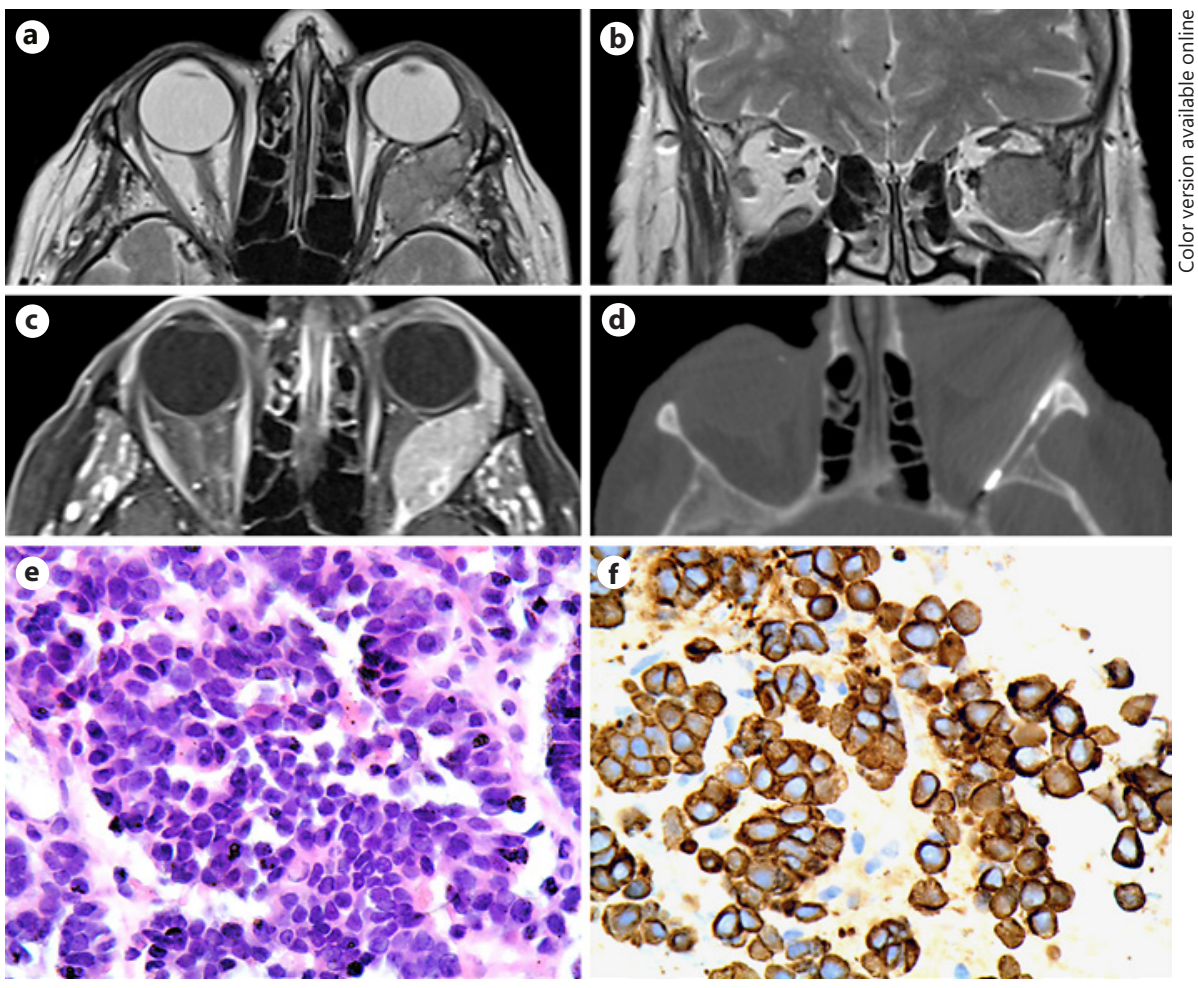

fibrous meningioma (grade 1) of the greater wing of sphenoid. All patients were kept in the hospital for at least $4 \mathrm{~h}$ to monitor for retrobulbar haemorrhage, but none of them developed any complications related to the procedure other than peri-ocular bruising. Further details on each of the 5 cases can be found in the supplementary material (for all online suppl. material, see www.karger. com/doi/10.1159/000510867).

\section{Discussion}

Our study showed that CT-CNB of inflammatory and neoplastic orbital lesions can be an alternative to open biopsy in selected cases. In 4 out of 5 patients, the tissue sample acquired was enough to obtain a histopathological diagnosis. None of the 5 patients developed any local or systemic adverse events related to the procedure.

CNB under CT guidance for space-occupying lesions of the orbit was first described in 1996 by Alter et al. [14]. They reported its success in diagnosing 2 cases of intraorbital meningioma. Jeng Tyng et al. [15] reported the use of CT guidance to acquire a CNB in 2 patients who were eventually diagnosed as having schwannoma and melanocytoma, respectively. They used a trans-palpebral lateral approach comparable to our approach.
CNB has also been performed blindly and under ultrasound guidance. Yarovoy et al. [13] reported its use on 50 orbital lesions in 46 patients. In 39, the lesion was palpable, and the biopsy was performed without image guidance. In the remaining 11, they used ultrasound guidance. Two of their patients developed retrobulbar haemorrhage. The orbital lesions in their study were relatively large and ranged from $19 \times 20 \times 25 \mathrm{~mm}$ to diffuse involvement of the orbit, which made them easy to localise. Orlandi et al. [12] reported on the use of ultrasound-guided $\mathrm{CNB}$ on 13 orbital lesions that ranged in size from 2.3 to $4.2 \mathrm{~cm}$; only 5 were retrobulbar. Retrobulbar haemorrhage developed in 1 of the patients.

The use of CT guidance has several advantages. It allows more accurate localisation of posteriorly located, impalpable, and smaller orbital lesions, while at the same time minimizes inadvertent injury to orbital structures. In Case 5 in our study, accurate localisation of the lesion was particularly useful when the biopsy showed an inflammatory process; this eliminated the possibility of the sample being superficial and not reflecting the true pathology. Despite such an advantage, we failed to acquire an adequate tissue sample in Case 3. We attributed this to the fact the tumour originated within a bony structure, i.e., the greater wing of sphenoid bone, and the calcified nature of the mass. In retrospect, we believe that, using a different

Ocul Oncol Pathol 2021;7:54-61

DOI: $10.1159 / 000510867$ 
needle such as the Jamshidi, Craig, or Michele needles, we might have been able to obtain an adequate sample; all of these alternatives have been shown to be effective in obtaining samples from tumours within bony structures [16, 17]. However, these needles are significantly larger, have a wider diameter, and require greater force to advance them into bony lesions. Their use would likely be associated with a significantly increased rate of complications. A direct lateral orbital approach would be a safer choice.

Another advantage of CT guidance is that it enables the early detection of retrobulbar haemorrhage when performing the biopsy, since blood can easily be identified on non-contrast CT scan and then acted upon quickly.

CT guidance has also been used with FNA $[3,18]$. However, CNB has a significant advantage over FNA. FNA is only suitable for cytological analysis as it provides a small sample and distorts the tissue architecture. It is less reliable than $\mathrm{CNB}$ for specific tissue diagnosis, differentiating malignant and benign lesions, and tumour classification and grading. Krohel et al. [7] showed that FNA performed under direct visualisation during open biopsy was either inadequate or inaccurate for making a diagnosis in 53\% of their patients. Similarly, Karcioglu et al. [19] showed a low agreement rate between histopathological and cytopathological diagnoses. They obtained the FNA under direct visualisation using an in vitro technique. The rate of agreement was lower for primary malignant orbital tumours, like lymphomas, and for benign orbital tumours. For metastatic tumours and inflammatory disorders, the cytopathological and histopathological diagnoses matched in all patients. Wiktorin et al. [20] showed a higher success rate $(87 \%)$ in obtaining a histological diagnosis using FNA, but this was significantly less likely to be successful for impalpable versus palpable lesions (75 vs. $90 \%$ ). Also, while the FNA diagnosis correlated with the final diagnosis in $87 \%$ of their patients, $46 \%$ still required further incisional and excisional biopsies.

We have found CNB to be useful mainly in cases where surgical excision or debulking of the mass lesion is not indicated such as metastatic tumours, tumours sensitive to chemotherapy or radiotherapy, and inflammatory disorders. It does not require general anaesthesia, which gives it an advantage over open biopsy, particularly in elderly and severely ill patients. It also allows for quicker delivery of radiotherapy in radiosensitive tumours, compared to open biopsy in which wound-healing may take several weeks. CNB is quicker and cheaper than open biopsy and allows allocation of operating-theatre time to other patients on the waiting list. A lateral orbitotomy on such a patient would require $3 \mathrm{~h}$ of theatre time; with run- ning costs averaging approximately GBP $1,200 / \mathrm{h}$, this surgery would thus cost more than GBP 3,600. The patient would have to stay overnight after surgery (another GBP 400), incurring a total cost in excess of GBP 4,000. On the other hand, we estimate the cost of CT-CNB to be around GBP 1,000. This includes the cost of using the CT scanner suite for $1 \mathrm{~h}$ (GBP 300), the cost of a day case procedure (GBP 700), and the cost of having 1 ophthalmologist and 2 radiologists perform the procedure.

Nevertheless, this procedure carries several risks. A peribulbar block can induce oculo-cardiac reflex and death. Thus, it is important that resuscitation equipment and medical personnel capable of delivering cardiopulmonary resuscitation are available at the site of the procedure. The risks also include retrobulbar haemorrhage, and inadvertent globe and/or optic nerve injury. The use of CT guidance significantly reduces such risks, as demonstrated by the fact that none of our patients developed any complications, but it does not completely eliminate them. To minimize the risk of bleeding, anti-coagulation should be managed as per open biopsy. This includes discontinuation of the anti-coagulants and bridging the patient with heparin, when indicated, after liaison with the treating physicians and a haematologist when further advice is needed. It is also important that an ophthalmologist is available to deliver the peribulbar block and perform a canthotomy and cantholysis in the event of a bleed. In all our patients, the lateral canthal area was anaesthetised to allow the passage of the CNB needle; this would also have saved time in the event of canthotomy and cantholysis being required. It is advisable to monitor the patient for at least $4 \mathrm{~h}$ following the procedure in case of a delayed retrobulbar haemorrhage. Total akinesia is imperative, to lower the risk of inadvertent injury to the orbital structures and ensure stable orbital anatomy throughout the procedure. The use of CT exposes the patient to a large amount of ionizing radiation; however, our technique uses the least radiation possible and limits the scan to the level on which the biopsy is performed.

Due to the abovementioned risks, it is imperative that the 2 interventional radiologists performing the procedure are very familiar with the orbital anatomy and welltrained to perform CNB. It is essential for an orbital surgeon to be present and ensure the procedure is done safely until the interventional radiologists become more familiar with the procedure. It is also important that the procedure is not performed on vascular lesions as these would bleed profusely, and also when the mass lesion is in close proximity to vital structures.
60

Ocul Oncol Pathol 2021;7:54-61 DOI: $10.1159 / 000510867$
Bata/Martin/Connolly/Mudhar/Hersey/ Salvi 
Despite the promising outcome, our study has several limitations. The number of patients was small and did not represent all the different aetiologies of space-occupying lesions of the orbit. All tumours were located in the lateral or supero-lateral aspect of the orbit, so this technique may not be possible for mass lesions located elsewhere within the orbit, or else a modification to the technique would be needed. Also, while CNB may hold a theoretical advantage over FNA, a direct comparison is needed before concluding whether this theoretical advantage is true or not.

In conclusion, CT-CNB of soft-tissue inflammatory and neoplastic lesions located in the lateral aspect of the orbit could be an alternative to obtain tissue samples for histopathological diagnosis, in selected cases when an open flap biopsy is not possible, particularly in patients for whom general anaesthesia carries a high risk and when no further surgery is anticipated. It is more likely to provide an adequate sample than FNA. Nevertheless, larger studies are required to examine its safety and effectiveness.

\section{Statement of Ethics}

The study adhered to the tenets of the Declaration of Helsinki. All subjects were aware of the limited evidence on the use of this technique. They all signed a written consent that stated in the event of a failure to acquire an adequate tissue sample, an orbitotomy would be required. A written informed consent to publish her photos was obtained from the patient whose external photos feature in Figure 1. Neither the text nor the figures contain any identifiers of the remaining 4 patients. This study was approved as a clinical effectiveness project by our institute (ethics approval No. CEU 9430).

\section{Conflict of Interest Statement}

The authors have no conflicts of interest to declare.

\section{Funding Sources}

This study was not funded by any financial body.

\section{Author Contributions}

B.M.B designed the study, collected the data, wrote the manuscript, and prepared the figures. A.M. wrote the section on the biopsy technique and parts of the discussion, prepared the radiology figures, and revised the manuscript. D.C., N.H., and S.S. contributed to the idea of the procedure and the paper and revised the manuscript. H.S.M. wrote the histopathology sections and revised the manuscript.

\section{References}

1 Schyberg E. Fine needle biopsy of orbital tumours [proceedings]. Acta Ophthalmol Suppl. 1975; 125:11.

2 Kennerdell JS, Dekker A, Johnson BL, Dubois PJ. Fine-needle aspiration biopsy. Its use in orbital tumors. Arch Ophthalmol. 1979;97: 1315-17.

3 Dubois PJ, Kennerdell JS, Rosenbaum AE, Dekker A, Johnson BR, Swink CA. Computed tomographic localization for fine needle aspiration biopsy of orbital tumors. Radiology. 1979 Apr;131(1):149-52.

4 Tijl JW, Koornneef L. Fine needle aspiration biopsy in orbital tumours. Br J Ophthalmol. 1991 Aug;75(8):491-2.

5 Gupta S, Sood B, Gulati M, Takhtani D, Bapuraj R, Khandelwal N, et al. Orbital mass lesions: US-guided fine-needle aspiration biopsy-experience in 37 patients. Radiology. 1999 Nov;213(2):568-72.

6 Zajdela A, Vielh P, Schlienger P, Haye C. Fine-needle cytology of 292 palpable orbital and eyelid tumors. Am J Clin Pathol. 1990 Jan;93(1):100-4.

7 Krohel GB, Tobin DR, Chavis RM. Inaccuracy of fine needle aspiration biopsy. Ophthalmology. 1985 May;92(5):666-70.

8 Quinn SF, Nelson HA, Demlow TA. Thyroid biopsies: fine-needle aspiration biopsy versus spring-activated core biopsy needle in $102 \mathrm{pa}$ tients. J Vasc Interv Radiol. 1994 Jul-Aug; 5(4):619-23.

9 Ballo MS, Sneige N. Can core needle biopsy replace fine-needle aspiration cytology in the diagnosis of palpable breast carcinoma. A comparative study of 124 women. Cancer. 1996 Aug;78(4):773-7.

10 Renshaw AA, Pinnar N. Comparison of thyroid fine-needle aspiration and core needle biopsy. Am J Clin Pathol. 2007 Sep;128(3): 370-4.

11 Chowdhury T, Barnacle A, Haque S, Sebire N, Gibson S, Anderson J, et al. Ultrasound-guided core needle biopsy for the diagnosis of rhabdomyosarcoma in childhood. Pediatr Blood Cancer. 2009 Sep;53(3):356-60.

12 Orlandi D, Sconfienza LM, Lacelli F, Bertolotto M, Sola S, Mauri G, et al. Ultrasound-guided core-needle biopsy of extra-ocular orbital lesions. Eur Radiol. 2013 Jul;23(7):1919-24.

13 Yarovoy AA, Bulgakova ES, Shatskikh AV, Uzunyan DG, Kleyankina SS, Golubeva OV. CORE needle biopsy of orbital tumors. Graefes Arch Clin Exp Ophthalmol. 2013;251(8): 2057-61.

14 Alter C, Heywang-Köbrunner SH, Beck R. [Diagnosis of intraorbital meningioma]. Aktuelle Radiol. 1996 Sep;6(5):232-4.
15 Jeng Tyng C, Matushita JP Jr, Bitencourt AG, Neves FB, Amoedo MK, Barbosa PN, et al. Uncommon primary tumors of the orbit diagnosed by computed tomography-guided core needle biopsy: report of two cases. Radiol Bras. 2014 Nov-Dec;47(6):380-3.

16 van der Bijl AE, Taminiau AH, Hermans J, Beerman H, Hogendoorn PC. Accuracy of the Jamshidi trocar biopsy in the diagnosis of bone tumors. Clin Orthop Relat Res. 1997 Jan;(334):233-43.

17 Moore TM, Meyers MH, Patzakis MJ, Terry R, Harvey JP Jr. Closed biopsy of musculoskeletal lesions. J Bone Joint Surg Am. 1979 Apr;61(3):375-80.

18 Kennerdell JS, Dubois PJ, Dekker A, Johnson BL. CT-guided fine needle aspiration biopsy of orbital optic nerve tumors. Ophthalmology. 1980 Jun;87(6):491-6.

19 Karcioglu ZA, Fleming JC, Haik BG. A prospective comparison of fine-needle aspiration cytopathology and histopathology in the diagnosis of orbital mass lesions. Br J Ophthalmol. 2010 Jan;94(1):128-30.

20 Wiktorin AC, Dafgård Kopp EM, Tani E, Söderén B, Allen RC. Fine-Needle Aspiration Biopsy in Orbital Lesions: A Retrospective Study of 225 Cases. Am J Ophthalmol. 2016 Jun;166:37-42. 